\title{
Spontaneous dormancy of metastatic breast cancer cells in an all human liver microphysiologic system
}

\author{
S E Wheeler ${ }^{1,9}$, A M Clark ${ }^{1,9}$, D P Taylor ${ }^{1,2}$, C L Young ${ }^{3}$, V C Pillai ${ }^{4}$, D B Stolz ${ }^{1,5,6,7}$, R Venkataramanan ${ }^{1,4}$, \\ D Lauffenburger ${ }^{3}$, L Griffith ${ }^{3}$ and A Wells L $^{*}, 2,6,7,8$
}

${ }^{1}$ Department of Pathology, University of Pittsburgh, S711 Scaife Hall, 3550 Terrace Street, Pittsburgh, PA, USA; ${ }^{2}$ Department of Bioengineering, University of Pittsburgh, Pittsburgh, PA, USA; ${ }^{3}$ Department of Biological Engineering, Massachusetts Institute of Technology, Cambridge, MA, USA; ${ }^{4}$ Department of Pharmaceutical Sciences, University of Pittsburgh, Pittsburgh, PA, USA; ${ }^{5}$ Department of Cell Biology, University of Pittsburgh, Pittsburgh, PA, USA; ${ }^{6}$ McGowan Institute for Regenerative Medicine, University of Pittsburgh, Pittsburgh, PA, USA; ${ }^{7}$ University of Pittsburgh Cancer Institute, Pittsburgh, PA, USA and ${ }^{8}$ Pittsburgh VA Medical Center, VA Pittsburgh Healthcare System, Pittsburgh, PA, USA

Background: Metastatic outgrowth in breast cancer can occur years after a seeming cure. Existing model systems of dormancy are limited as they do not recapitulate human metastatic dormancy without exogenous manipulations and are unable to query early events of micrometastases.

Methods: Here, we describe a human ex vivo hepatic microphysiologic system. The system is established with fresh human hepatocytes and non-parenchymal cells (NPCs) creating a microenvironment into which breast cancer cells (MCF7 and MDA-MB-231) are added.

Results: The hepatic tissue maintains function through 15 days as verified by liver-specific protein production and drug metabolism assays. The NPCs form an integral part of the hepatic niche, demonstrated within the system through their participation in differential signalling cascades and cancer cell outcomes. Breast cancer cells intercalate into the hepatic niche without interfering with hepatocyte function. Examination of cancer cells demonstrated that a significant subset enter a quiescent state of dormancy as shown by lack of cell cycling ( $\mathrm{EdU}^{-}$or Ki67 ${ }^{-}$). The presence of NPCs altered the cancer cell fraction entering quiescence, and lead to differential cytokine profiles in the microenvironment effluent.

Conclusions: These findings establish the liver microphysiologic system as a relevant model for the study of breast cancer metastases and entry into dormancy.

One in every eight women in the United States will be diagnosed with breast cancer in her lifetime. Over one-third of these women will experience local or distant disease spread. Distant metastasis is the major cause of breast cancer-related mortality with a 5-year survival rate of $20 \%$ after detection of a clinically overt metastatic nodule or site. Most daunting to women and men with breast cancer is that half of the disseminated disease emerges clinically after 5 or more years of seeming 'cure' from removal of the primary tumour (Fisher et al, 2002; Demicheli et al, 2005). Couple this with the fact that distant metastases are often resistant to standard treatments (Perloff et al, 1978; Gonzalez-Angulo et al, 2007), clearly underscores the need to better understand the molecular and cellular bases of metastatic seeding and entry into dormancy (Wells et al, 2013).

Metastasis is a multistep process whereby cells from the primary tumour invade the surrounding tissues and eventually disseminate

\footnotetext{
*Correspondence: Dr A Wells; E-mail: wellsa@upmc.edu

${ }^{9}$ These authors contributed equally to this work.
}

Received 1 July 2014; revised 8 September 2014; accepted 11 September 2014; published online 14 October 2014 
through the vasculature or lymphatic systems. Once disseminated these breast cancer cells extravasate into metastatic niches (Micalizzi et al, 2010). Survival and proliferation in the new metastatic niche is the most rate-limiting step in the formation of macrometastases (Luzzi et al, 1998; Cameron et al, 2000). The common pattern of metastasis in breast cancer is to the bone marrow, liver, lung, and brain, suggesting that metastases require organs that provide the necessary microenvironment (Fidler, 1970; Viadana et al, 1973). With respect to breast cancer, extirpation of the primary mass can initially provide a seeming cure with clinical metastatic outgrowth delayed many years before emergence. This phenomenon of latent outgrowth could be due to balanced growth and proliferation or dormancy. Recent work in our laboratory has demonstrated in silico that a quiescent state is far more likely than balancing proliferation and apoptosis to remain subclinical over extended periods (Taylor et al, 2013); this concurs with findings of solitary tumour cells or small clusters having low mitogenic rates in animals (Ghajar et al, 2013) and patients (Bednarz-Knoll et al, 2011). The mechanism of entry into dormancy may integrate with recent findings of phenotypic plasticity in which highly dedifferentiated aggressive breast cancers appear more epithelial in the metastatic niche (Bukholm et al, 2000; Kowalski et al, 2003; Chao et al, 2010, 2012), and suggest that a reversion of the epithelial-tomesenchymal transition that presages dissemination of the primary tumour has a major role in breast cancer dormancy (Gunasinghe et al, 2012). Thus, we sought a model system that could yield quiescent metastatic breast cancer cells.

The occurrence of micrometastasis that can remain dormant for years before re-emerging as a full metastasis represents a major unsolved clinical problem, particularly in breast cancer, and how such metastases resist chemotherapy and then re-emerge remains poorly understood. Because single cancer cells are difficult to detect, little is known about the cell dynamics of single disseminated breast cancer cell seeding and the ensuing dormancy. Emergence and subsequent outgrowth after dormancy has been more widely studied in recent years but the requirements for tumour cells to seed and remain clinically undetectable in a metastatic niche are unknown. Difficulties in studying micrometastases arise due to the limitations of current models. Animal models typically only provide endpoint analyses, which are compounded by issues of relevance to the human condition (Mestas and Hughes, 2004; Hackam and Redelmeier, 2006). Such studies also predominantly utilise immunocompromised murine models (Khanna and Hunter, 2005; Fantozzi and Christofori, 2006; Teicher, 2006; Ghajar et al, 2013) even though the immune system is well established in having an important role in the metastatic microenvironment (Junttila and de Sauvage, 2013; Slaney et al, 2013). As a result, research is turning towards $3 \mathrm{D}$ organotypic cultures in bioreactors as exploratory tools to overcome such issues (Shaw et al, 2004; Nelson et al, 2006; Gomez et al, 2010; Ridky et al, 2010; Stroock and Fischbach, 2010; Domenech et al, 2012; Weigelt et al, 2014). Our all-human microphysiologic system uses fresh human tissue to recapitulate the hepatic microenvironment and allow for spontaneous quiescence in a population of MDA-MB-231 breast cancer cells in an all-human target organoid (Yates et al, 2007).

\section{MATERIALS AND METHODS}

Fresh human hepatocytes and non-parenchymal cells. The hepatocytes (hHep) and non-parenchymal cells (NPCs) for this work are excess pathological specimens from therapeutic partial hepatectomies for metastatic colorectal carcinoma or other benign diseases such as focal nodular hyperplasia. These cells are available from the NIDDK-funded Liver Tissue and Cell Distribution System (LTCDS) with the procurement core directed by Dr David Geller at the University of Pittsburgh, which was funded by NIH
Contract \# HHSN276201200017C. Through this distribution system the livers are perfused and separate isolations of hHep and NPCs are provided to investigators. We further purified the NPC fraction (to eliminate contaminating debris, hepatocytes, and red blood cells) as previously reported (Hwa et al, 2007).

Cell lines. MDA-MB-231 and MCF7 breast cancer cell lines were purchased from ATCC (Manassas, VA, USA) and transfected with red fluorescent protein (RFP) as previously described (Chao et al, 2010). Cells were maintained in RPMI-1640 with $10 \%$ heatinactivated FBS and $25 \mathrm{IU} \mathrm{ml}^{-1}$ penicillin and streptomycin (Gibco, Life Technologies, Grand Island, NY, USA). For seeding into the ex vivo hepatic microphysiologic system, cells were trypsinised and neutralised in complete growth medium, centrifuged and resuspended in hepatocyte maintenance medium.

Ex vivo hepatic microphysiologic system. The ex vivo hepatic microphysiologic system (LiverChip) is assembled as recommended by the manufacturer ( $\mathrm{CN}$ Bio Innovations Limited, Oxford, UK). Scaffolds for tissue growth are freshly coated with $1 \%$ rat tail collagen type I (BD Biosciences, Life Technologies, Grand Island, NY, USA) in PBS for $1 \mathrm{~h}$ at room temperature and washed with PBS before placement in the system. The ex vivo hepatic microphysiologic system is passivated with $1 \%$ BSA at $37^{\circ} \mathrm{C}$, which is then replaced with William's Medium E (Gibco, Life Technologies) supplemented with the Hepatocyte Thawing and Plating Supplement Pack (Life Technologies), prepared as recommended by the manufacturer. Hepatocytes and NPCs are plated at a ratio of $1: 1\left(6 \times 10^{5}\right.$ cells per well $)$ in the system, approximating physiologic ratios. Hepatocytes alone and in all coculture conditions were cultured for $\sim 16 \mathrm{~h}$ in the aforementioned media before changing to William's Medium E supplemented with the Hepatocyte Maintenance Supplement Pack (Life Technologies), prepared as recommended by the manufacturer. The medium is exchanged completely every $48 \mathrm{~h}$ and supernatant collected and immediately stored at $-80^{\circ} \mathrm{C}$ in cryogenic vials (Corning Life Sciences, Tewksbury, MA, USA) for downstream assays. Day 3 is considered as the first day of complete tissue formation and on this day cancer cells are introduced into the formed liver tissue (experimental overview, see Supplementary Figure 1).

Enzyme-linked immunosorbent assays. Alpha-1 antitrypsin (A1AT) and fibrinogen secretion from hepatocytes were measured using ELISA kits according to the manufacturer's instructions (Genway Biotech Inc., San Diego, CA, USA). Supernatants were used at a 1:150 dilution for A1AT and a 1:25 dilution for fibrinogen.

Clinical chemistry assays. Assays for glucose (GLU), blood urea nitrogen (BUN), aspartate transaminase (AST), and alanine aminotransferase (ALT) were performed in the College of American Pathologists certified clinical laboratories in the University of Pittsburgh Medical Center (UPMC, Pittsburgh, PA, USA) in accordance with all governmental regulations.

Immunofluorescence microscopy. Scaffolds were harvested and fixed in 2\% paraformaldehyde in PBS for $1 \mathrm{~h}$. Scaffolds rinsed three times in PBS, then blocked in PBS containing 0.5\% BSA with $0.5 \%$ normal goat serum for $30 \mathrm{~min}$ at room temperature. Primary antibodies (see list below) diluted in $0.5 \%$ BSA in PBS (PPB) buffer were added to sections $1 \mathrm{~h}$ at room temperature. Sections were washed five times in PBB Buffer then fluorescently tagged secondary antibodies (list below), diluted in PBB buffer, were added to the sections for $1 \mathrm{~h}$ at room temperature. Scaffolds were washed three times in PPB buffer, three times in PBS. Confocal images and stacks were obtained on an inverted Fluoview1000 confocal microscope (Olympus America Inc., Center Valley, PA, USA) using a $\times 10$ (UPlanApo NA=0.4) or $\times 20$ (UPlanSApo $\mathrm{NA}=0.85$ ) objective. Scaffolds were submerged in PBS in the coverslip well of a 35-mm MatTek glass bottomed dish 
(MatTek, Ashland, MA, USA) before imaging. Stacks were reconstructed using the MetaMorph Image analysis software (Molecular Devices, LLC, Sunnyvale, CA, USA). The primary antibodies used were mouse anti-human CD68 (KP-1, Dako Cytomation, Carpinteria, CA, USA), rabbit anti-human LYVE-1 (ab14917, Abcam, Cambridge, MA, USA), mouse anti-human CD45 (555480, BD Biosciences, San Jose, CA), and rabbit ant-Ki67 (Abcam, ab15580). Secondary antibodies used were goat antimouse or goat anti-rabbit Cy3, Cy5 (Jackson Immunoresearch, West Grove, PA, USA) or Alexa 488 (Invitrogen, Grand Island, NY, USA). Counterstains used were $0.1 \%$ Hoechst 33258 dye (Sigma, St Louis, MO, USA) for nuclei and Alexa 488 or Alexa 647 Phalloidin (Invitrogen) for F-actin.

Click-iT EdU proliferation assay. Proliferation was assessed using the Click-iT EdU Alexa Fluor 488 Imaging Kit (Invitrogen, Life Technologies) and was performed according to the manufacturer's instructions. Briefly, $20 \mu \mathrm{M}$ EdU was added on days 11 and 13 with the complete medium change before harvest on day 15 ( $96 \mathrm{~h}$ of continuous EdU exposure). EdU was then detected in those scaffolds containing EdU labelled tissue and cancer cells according to the manufacturer's instructions.

Cancer cell detection and enumeration. Images of the tissue containing scaffolds were taken using an Olympus BX51 with a $\times 2$ (PlanApo $\mathrm{NA}=0.08$ ) objective (Olympus America Inc.). Digital images were obtained on an Olympus CCD camera using the Magnafire image acquisition software (Olympus America Inc.). The level of RFP-expressing breast cancer cells was determined using the MetaMorph software (Molecular Devices, LLC). Images of the entire scaffolds were inclusively thresholded and the RFPpositive portion measured as a percentage of total scaffold area.

Cytochrome P450 assay. Various Cytochrome P450 (CYP) enzyme activities were measured using a previously validated CYP cocktail assay (Pillai et al, 2013). Cytochrome P450 cocktail substrates used in this assay were selected based on the FDA guidance over the preferred and acceptable CYP substrates (http:// www.fda.gov/drugs/developmentapprovalprocess/developmentresources/druginteractionslabeling/ucm093664.htm) for evaluating the CYP activities in vitro. Further, the validated five probes CYP cocktail assay was reported to have no significant intersubstrate interactions.

Luminex multiplex immunoassays. Determination of 56 cytokines, chemokines, and growth factors was performed using human group 127 -plex, cancer panels 1 and 2 according to the manufacturer's recommendations (Bio-Rad Laboratories, Inc., Hercules, CA, USA), with the exception that coupled beads, biotinylated detection antibodies, and streptavidin-phycoerythrin fluorescent reporters were diluted two-fold. All samples were diluted two-fold to attain measurements within the linear dynamic range of the assay; a total volume of $50 \mu$ l diluted sample was analysed per multiplex panel. Prepared arrays were assessed by the Bio-Plex 3D Suspension Array System (Bio-Rad Laboratories, Inc.) utilising xMAP technology licensed by Luminex. Data were collected with xPONENT for FLEXMAP 3D software, version 4.2 (Luminex Corporation, Austin, TX, USA).

Statistics. Mann-Whitney non-parametric tests or Student's $t$-tests were used unless otherwise specified. Graphs and statistics were generated using GraphPad Prism version 6 (GraphPad Software, Inc., La Jolla, CA, USA).

\section{RESULTS}

Ex-vivo systems to study metastasis are desirable as they allow for interrogation of the process, where in vivo experiments are more limited to endpoint analyses. We chose the hepatic niche as the metastatic site to study as the liver is a common site of cancer metastasis, liver ex vivo microphysiologic systems are advanced and the liver is the primary site of chemotherapy metabolism. Here, we began with the ex vivo hepatic microphysiologic system designed to maintain hepatocytes for weeks of culture in a 3D tissue like structure (Powers et al, 2002; Yates et al, 2007; Griffith et al, 2013).

Hepatocytes and NPCs survive in the ex vivo hepatic microphysiologic system. Non-parenchymal cells have been well established as important components of the liver, accounting for up to $40 \%$ of the cells in the liver (Crispe, 2009). Many microenvironmental approaches to liver metastasis incorporate only hepatocytes and possibly one other cell type (generally Kupffer, endothelial, or stellate cells). As a first step, we incorporated a crucial immune element, demonstrating that commercially available cryopreserved hepatocytes can form the tissue with Kupffer cells with both surviving 15 days of culture (Figure 1A); this approach is readily transferrable to other laboratories due to the availability of the cell sources. To more fully recreate the liver microenvironment, we recombined the NPC fraction from fresh liver resections with freshly isolated primary hepatocytes. Introducing hepatocytes and the NPC fraction in reflective proportions, we found that the NPCs survived the full culture period (Figure 1B) with Kupffer cells $\left(\mathrm{CD}^{+} 8^{+}\right)$, leukocytes $\left(\mathrm{CD} 45^{+}\right)$, and sinusoidal endothelial cells (Lyve- $1^{+}$) all identified. Day 13 stimulation of freshly isolated human hepatocytes and NPCs with LPS enhanced the production of the hepatocyte-specific acute-phase markers A1AT and fibrinogen (Supplementary Figure 2). This enhancement indicates that the NPC fraction is still functional through day 15.

Hepatocytes function in the ex vivo hepatic microphysiologic system. Having established survival of hepatocytes and NPCs in extended (15-day culture), we then characterised phenotypic functions over the time course of culture; this is critical as functioning wanes in $2 \mathrm{D}$ and sandwich cultures. We and others have found that hepatocytes undergo a transient adjustment to culture conditions following isolation, characterised by dying off of injured cells and recovery of hepatocellular gene expression as extracellular matrix accumulates and cell-cell junctions are strengthened. Hepatocyte injury was measured by the standard clinical markers of AST and ALT. These markers decreased as tissue formation matured in the reactor (Figure 1C); during the initial reorganisation, the injury markers are high but as cells adjust to culture and cells injured during isolation are eliminated or recover, AST and ALT drop to near undetectable levels. A similar trend is observed with A1AT and fibrinogen, two acute-phase proteins that show elevated expression in blood during inflammation but are normally produced uniquely by hepatocytes at low basal levels to control tissue remodelling and clotting. As expected, production of A1AT was initially high, and decreased throughout the culture period to a low homeostatic level at day (Figure 1D). Fibrinogen showed high donor-to-donor variability and no significant change in production through the culture period (Figure 1D). Glucose levels remained at the input level (Figure 1E), but the every 2-day replacement of the media containing $200 \mathrm{mg} \mathrm{dl}^{-1}$ glucose likely masks consumption by the cells. Urea, a by-product of protein catabolism that can also be elevated transiently during the initial culture, decreased through the culture period but was still detectable at day 15 (Figure 1F). These decreases during the 15-day period were expected as the initial 'injury' of seeding the bioreactor vessels subsides. We did not observe appreciable differences between hepatocyte alone and cultures with NPCs. We did not expect that the addition of NPCs would significantly alter hepatocyte function.

A critical measure of hepatocellular function is the ability to detoxify xenobiotics. The activities of four CYP enzymes were assessed on days 3 and 13 of culture. Of the CYP enzymes tested 
A

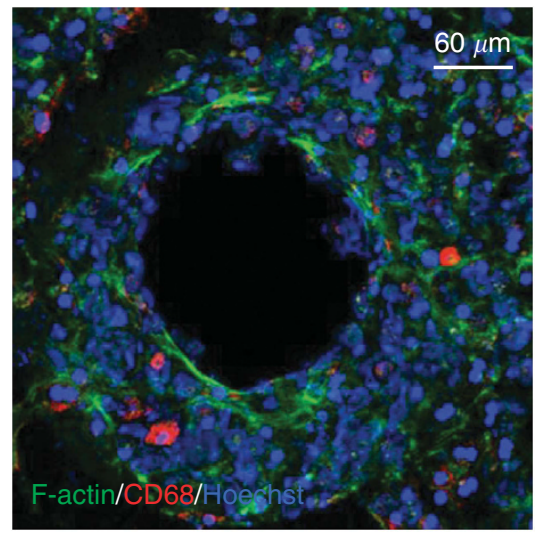

B

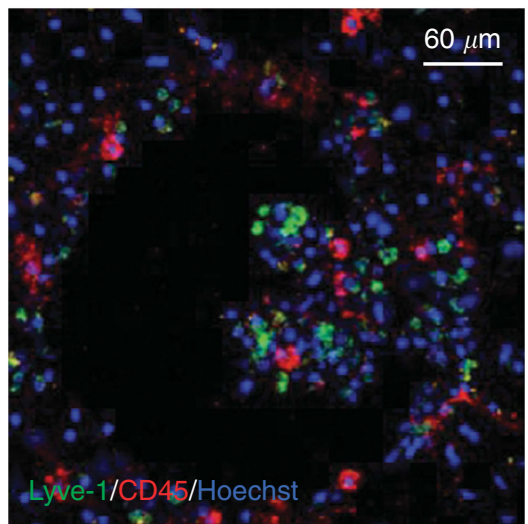

E

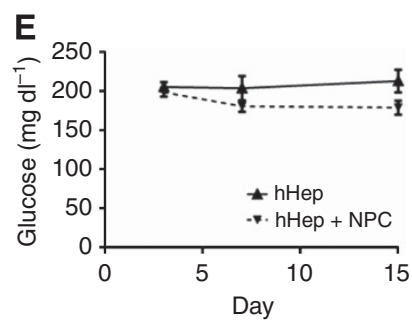

$\mathbf{F}$

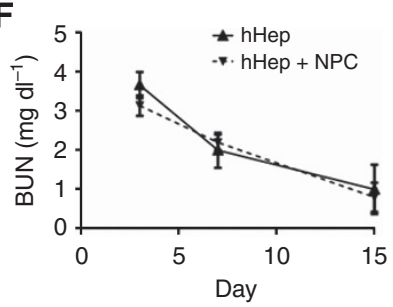

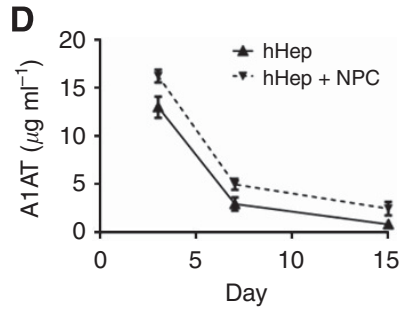
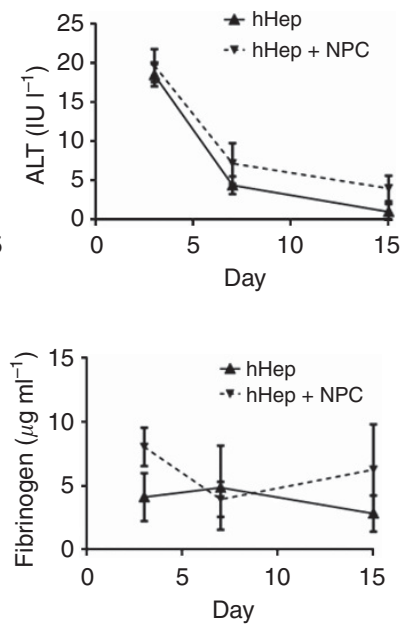

G

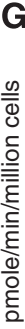

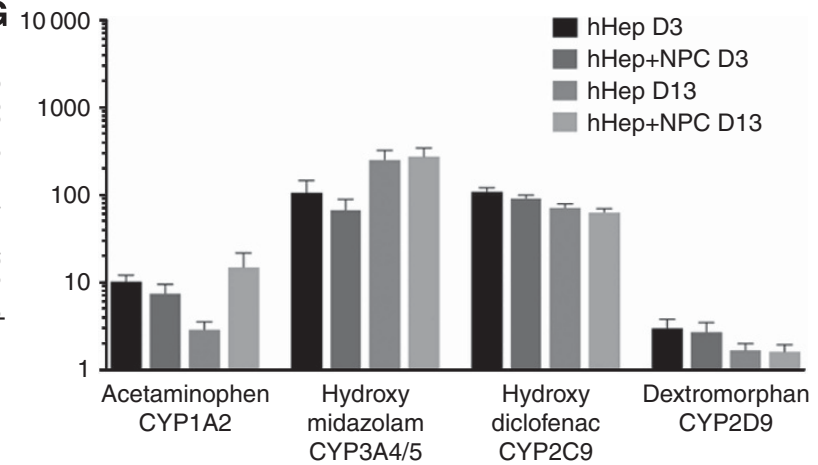

Figure 1. Hepatocytes and non-parenchymal cells survive and function through 15 days in the ex vivo hepatic microphysiologic system.

(A) Cryopreserved human hepatocytes were co-cultured with cryopreserved Kupffer cells through 15 days. CD68: red (macrophage marker), F-Actin: green, Hoechst: blue. (B) Freshly isolated human hepatocytes were seeded with a total NPC fraction from the same donor and cultured to 15 days. Lyve-1: green (liver sinusoidal endothelial cell marker), CD45: red (leukocyte marker), Hoechst: blue. (C-G) Freshly isolated human hepatocytes and NPC were seeded into the microphysiologic system and maintained with supernatant sampling through 15 days. (C) Hepatocyte injury was evaluated via clinical analyses of alanine aminotransferase $(P=0.0013)$ and aspartate aminotransferase $(P=0.0025)(n=5)$. (D) Protein production of alpha-1 anti-trypsin $(P<0.001)$ and fibrinogen $(P=0.09)$ by hepatocytes was measured by ELISA $(n=4)$. Statistical analyses are non-parametric Mann-Whitney tests comparing day 3 with day 15 for hHep + NPC. (E) Glucose consumption from the media (200 mg dl ${ }^{-1}$ ) was analysed via clinical tests for glucose concentration $(n=5 ; P=0.12)$. (F) Blood urea nitrogen was analysed with clinical tests to determine continued hepatocyte catabolism of proteins $(n=5 ; P=0.003)$. (G) Human hepatocytes with and without NPCs were assayed for cytochrome P450 activity on days 3 and 13 by LC-MS/MS for drug metabolites. CYP2D6 was sampled at $6 \mathrm{~h}$, all others sampled at $1 \mathrm{~h}(n=5)$. Statistical analyses are non-parametric Mann-Whitney tests comparing day 3 with day 15 for hHep + NPC.

there was little change in probe metabolism between days 3 and 13. CYP3A4/5 did show a significant increase between days 3 and 13 when hepatocytes were co-cultured with NPCs (Figure 1G). CYP2C9 showed a slight decrease in activity between days 3 and 13 when hepatocytes and NPCs were cultured together. Overall, there was low donor-to-donor variability for all markers except fibrinogen.
Breast cancer cell lines seed into the hepatic niche. To recapitulate the metastatic niche for breast cancer cells, hepatocytes and NPCs were allowed to form tissue for 3 days and MCF7 or MDA-MB-231 cells were seeded on day 3 and cultured through day 15 (12 days in the ex vivo hepatic microphysiologic system). Cancer cells were observed to successfully integrate with the 
hepatic tissue (Figure 2A). Confocal reconstruction demonstrates that the cancer cells did not merely seed on top of the tissue but had intercalated into the tissue residing alongside the hepatocytes (Figure 2B) as previously noted (Yates et al, 2007). Cancer cells were introduced into the system at a sufficiently low number to recapitulate metastases to the liver, without causing a stage 4 type (progressive growth) burden on the hepatic tissue (i.e., the ratio of liver cells to carcinoma cells to liver cells was >1000:1). Active CYP metabolism was evaluated on day 3 as a control and then on day 13 with and without cancer cells. Comparable activity was observed in CYP activity on day 13 following the introduction of cancer cells (Figure 2C). To ensure that the cancer cells did not induce injury in the hepatocytes, AST and ALT levels were measured with and without cancer cells and no change in the decreased injury with time was found (Figure 2D). Cancer cells being glycolytic, glucose levels were measured with and without cancer cells in the hepatic niche as a rough measurement of overburden by tumour cells. No significant changes in glucose levels were detected over the 15-day period (Figure 2E), again likely due to the large reservoir of glucose in the media and small number of tumour cells. Analysis of blood urea nitrogen, as a marker of overall cell function, demonstrated no difference between tissue with and without cancer cells (Figure 2F). We found that cancer cells integrated into the tissue without disrupting any of the probed
A

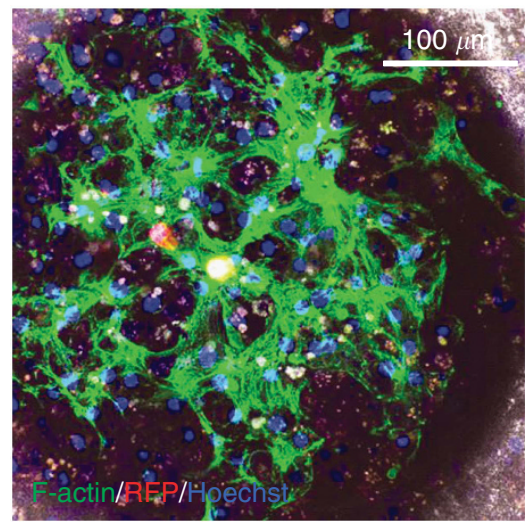

B

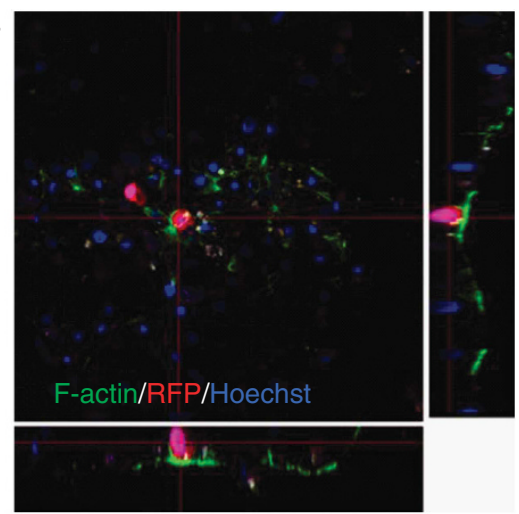

C

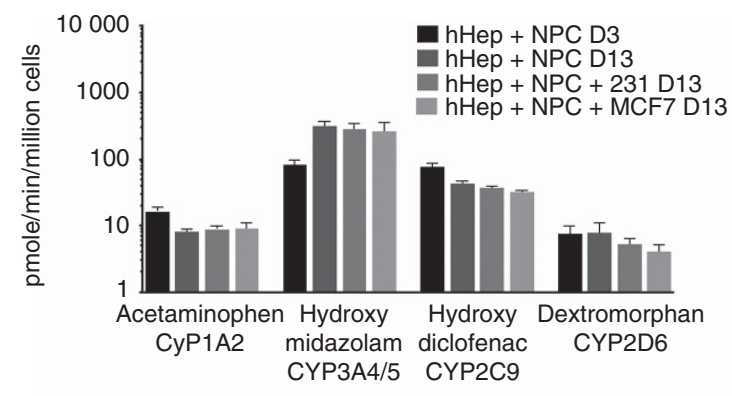

D
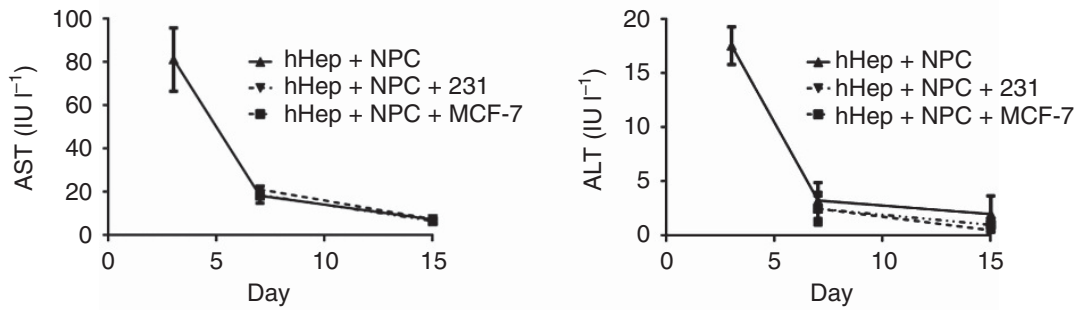

E
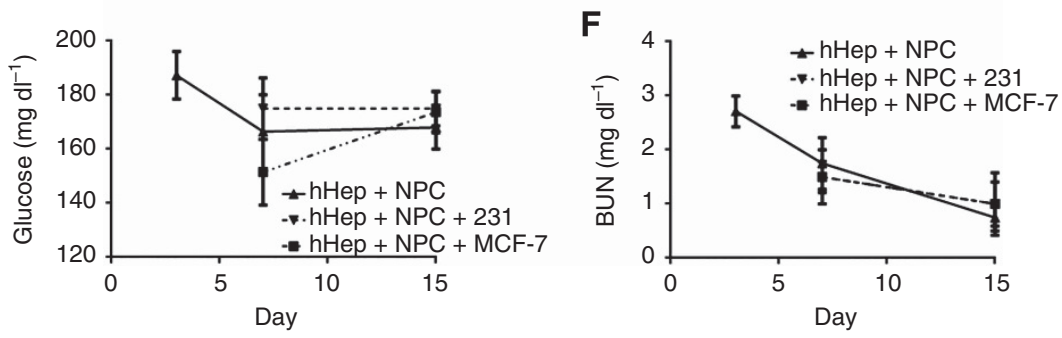

Figure 2. Breast cancer in the hepatic niche. Freshly isolated human hepatocytes and NPCs were seeded into the microphysiologic system and formed tissue by day 3 when MCF7 cells were introduced and cultures maintained through day 15. Scaffolds were harvested and fixed on day 15. (A) Tissue formation and morphology depicted by confocal reconstruction. Phase contrast is included for an outline of scaffold. MCF7: red, F-Actin: green Hoechst: blue. (B) Orthogonal plane composite of (A) to demonstrate tissue depth and intercalation of cancer cell. MCF7: red, F-Actin: green, Hoechst: blue. (C) Cytochrome P450 activity was measured by LC MC/MS of drug metabolites in cultures with and without cancer cells $(n=4)$. (D) Hepatocyte injury was evaluated via clinical analyses of alanine aminotransferase and aspartate aminotransferase $(n=4)$. (E) Glucose consumption from the media $\left(200 \mathrm{mg} \mathrm{dl}^{-1}\right)$ was analysed via clinical tests for glucose concentration $(n=4)$. ( $\left.F\right)$ Blood urea nitrogen was analysed with clinical tests to determine continued hepatocyte catabolism of proteins $(n=4)$. 
functions of the hepatic microenvironment. This is reflective of the human situation wherein micrometastases and even overt metastases do not induce hepatic dysfunction until well advanced.

Spontaneous growth attenuation in a sub-population of breast cancer cells. With the establishment of a more physiologic metastatic niche in this system, we observed, via confocal microscopy, spontaneous attenuation of cancer cell growth after day 15 in the ex vivo hepatic microphysiologic system (Figure 3 ). MCF7 cells, which express high levels of E-cadherin, demonstrated sub-populations of individual or 2-5 cell groups after an incubation period of 12 days (Figure 3A). MDA-MB-231 cells, which do not express appreciable levels of E-cadherin, could be anticipated to grow out rapidly and overtake the cultures. Confocal imaging of this cell line indicated that again, a sub-population of cells had spontaneously undergone growth attenuation (Figure 3A). However, the proportion was notably less than that observed for MCF7 cells. This growth attenuation was confirmed to be more than just individual cell migration through the absence of Ki67 staining and EdU incorporation in these cells (Figure 3B and C). After day 11 of culture, cells were treated with EdU for $96 \mathrm{~h}$ to track dividing cells. We found a sub-population of cells that had not undergone division in the last 4 days of culture (Figure 3C), with this being more common in the MCF7 cells. Quantification in two donors confirmed the trend towards decreased cell proliferation in MCF7 cells (Figure 3D). Notably, the observed attenuation was consistent across multiple donors (Supplementary Figure 3).

NPCs alter the tumour cell number and signalling of the hepatic microenvironment and cancer cells. NPCs comprise endothelial, stromal, and immune cells, which are known to have effects on the tumour microenvironment. We considered that the NPCs may have a profound effect on overall cancer cell burden compared with hepatocytes alone, either by allowing more survival or by driving proliferation. The presence of NPCs was associated with a decrease in the number of MDA-MB-231 cells but an increase in the MCF7 cells (Figure 4A and B); it should be noted that even with the increase levels the overall number of the epithelial MCF7 was still below that of the attenuated mesenchymal MDA-MB-231. However, there was significant donor-todonor variation, particularly in the E-cadherin-positive MCF7 cell line. The differential response between the cell lines was intriguing. We pursued the MDA-MB-231 cells further due to the more aggressive nature of the cell line and analysed supernatants from day 15 on the Luminex platform.

To determine the signalling contribution of NPCs specifically in recapitulating the metastatic microenvironment, we evaluated the effects of adding NPCs in the presence of cancer cell stimulus. The cytokines, chemokines, and growth factors analysed with NPC inclusion are depicted in Figure $4 \mathrm{C}$ and E. Elevations were observed in signals produced by endothelial (IP-10, PAI-1, angiopoietin-2, GM-CSF) and Kupffer cells (GM-CSF, MIP-1 $\beta$, IL-8) confirming their survival through 15 days in culture. This was further verified by elevations in acute-phase protein production (fibrinogen and A1AT) following LPS stimulation (Supplementary Figure 2). Other changes in signals involved in cancer attenuation or progression were observed. In the presence of NPCs, MDA-MB-231 cultures were associated increases in possible cancer attenuation signals (follistatin) and reductions in pro-inflammatory signals (IGFBP-1, MCP-1, MIP- $1 \alpha$, IL-6). Some cancer-associated signals were decreased (uPA, osteopontin, EGF), while others were increased (sHER-2) (Figure 4C). Conversely in MCF7 cultures, increases in possible cancer associated signals (osteopontin, sHER-2, VEGF-A, uPA, EGF) and pro-inflammatory signals (IL-6, MCP-1) predominated (Figure 4E). The ability to use these initial signalling molecules as prognostic or predictive biomarkers awaits much more extensive investigations.

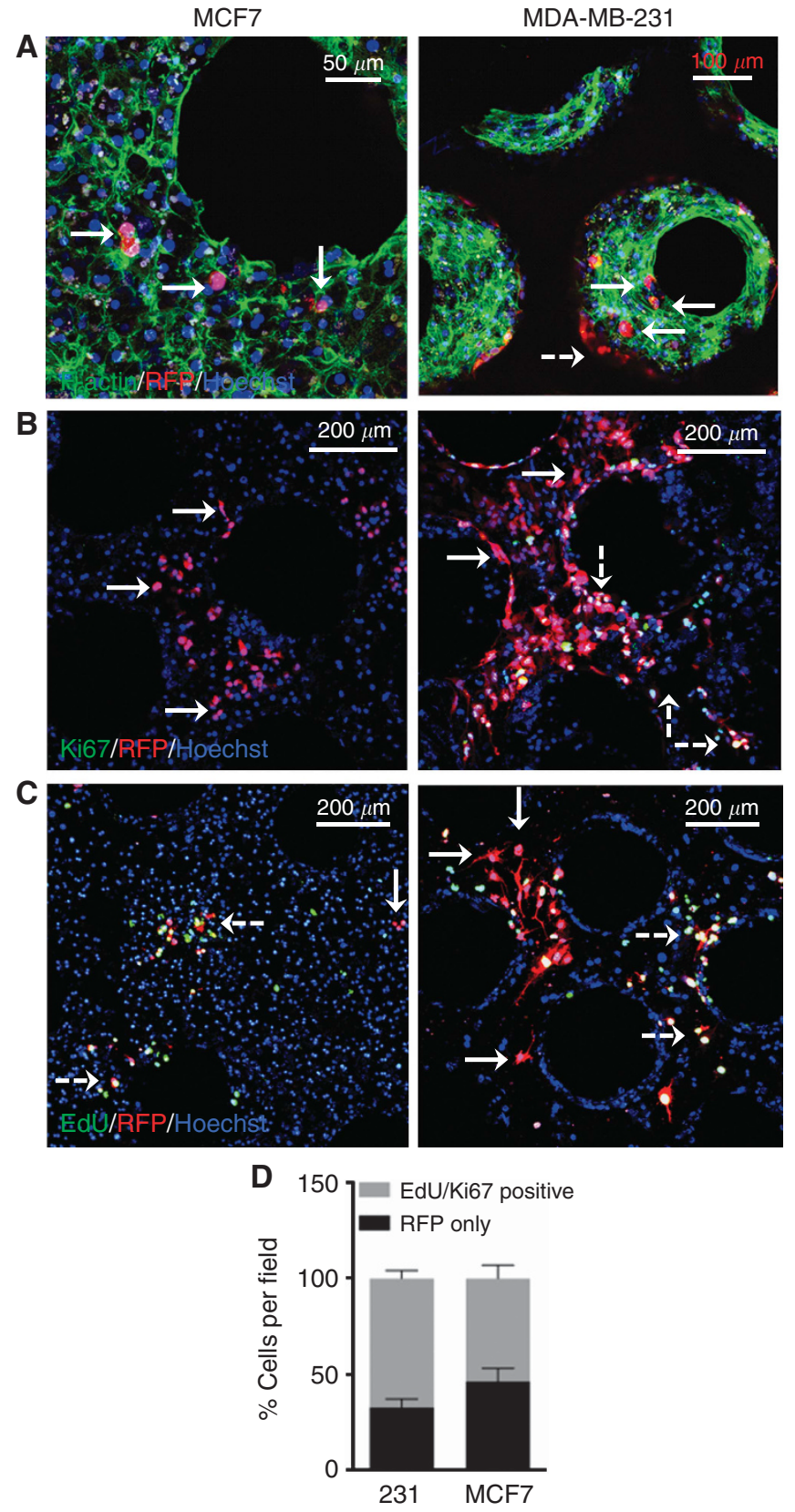

Figure 3. Spontaneous growth attenuation in a sub-population of breast cancer cell lines. (A) The E-cadherin expressing breast cancer cell line MCF7 (left; 1000 cells per well) and the mesenchymal cell line MDA-MB-231 (right; 100 cells per well) seeded on day 3 of hepatocyte and NPC culture and maintained through day 15 . Scaffolds were fixed and immunostained. Cancer cells: red, F-Actin: green, Hoechst: blue. (B) MCF7 and MDA-MB-231 breast cancer cell lines, seeded on day 3 of hepatocyte and NPC culture. Scaffolds were fixed and immunostained. Cancer cells: red, Ki67: green, Hoechst: blue. (C) On days 11 and 13 of culture, EdU $(20 \mu \mathrm{m})$ was added to fresh culture medium before harvesting scaffolds on day 15 ( $96 \mathrm{~h}$ for incorporation). Scaffolds were fixed and immunostained. Cancer cells: red, EdU: green, Hoechst: blue. Solid white arrow: attenuated cell, Dashed white arrow: proliferating cell. (D) Ki67 and EdU status was ennumerated for 6 fields per donor in two separate donors for both MCF7 and MDA MB 231 breast cancer cell lines. Status is expressed as percent total cancer cells as enumerated by RFP staining per imaging field.

Cross-talk between the metastatic microenvironment and cancer cells was investigated and cytokines, chemokines, and growth factors analysed are depicted in Figure 4D. In the presence 
A
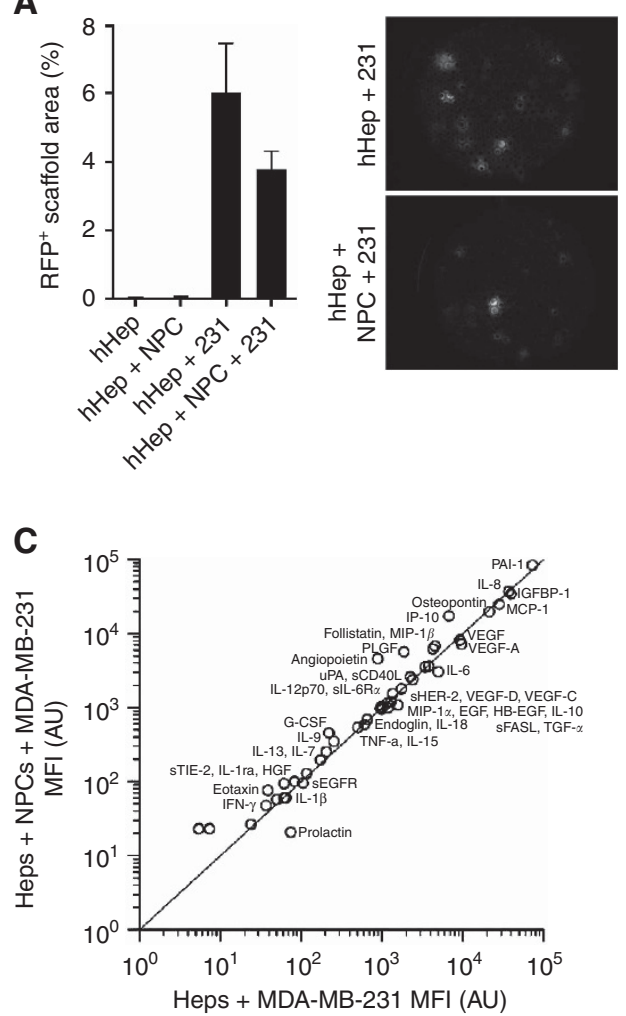

B
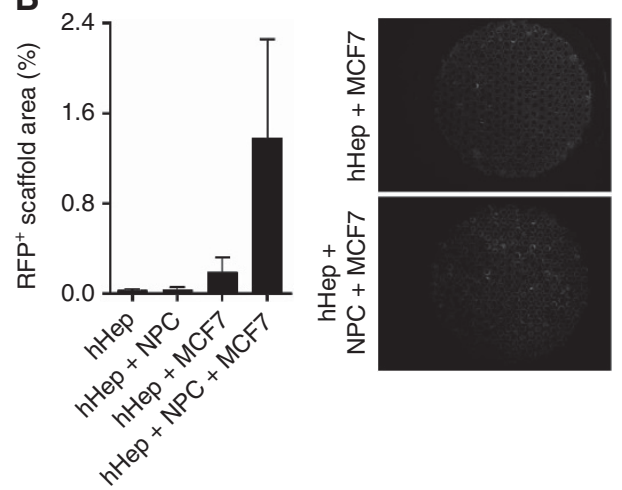

D

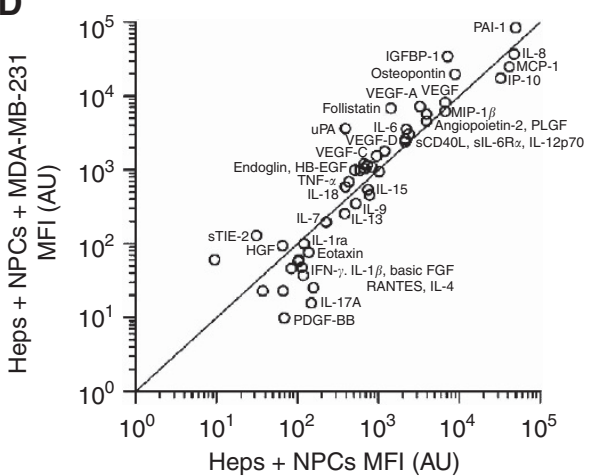

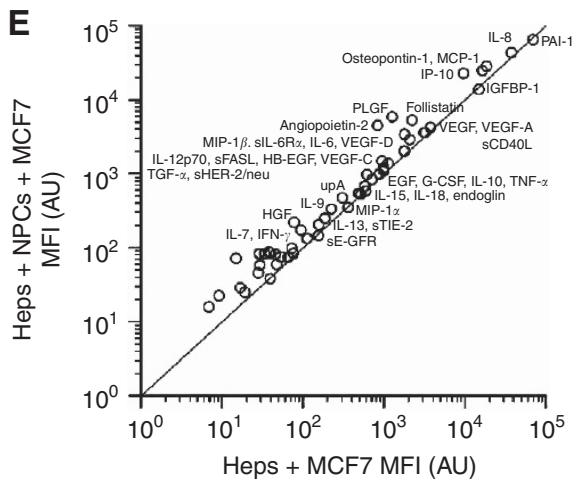

Figure 4. Non-parenchymal cells change the cell number and signalling of cancer cells in the metastatic niche. Hepatocytes and NPCs were plated to form tissue to day 3 when cancer cells were added. Cultures were maintained through day 15 with supernatant sampling. Scaffolds were harvested and fixed on day 15. (A, B) Complete scaffolds were imaged in the RFP channel ( $\times 2$ objective) and percent of total area that is RFP positive was calculated. Both MDA-MB-231 (A) and MCF7 (B) were quantified and plotted $(n=8)$. (C, D) Scatter plots of hepatocytes and MDAMB-231 co-cultures \pm NPCs (C) or hepatocytes and NPC co-cultures \pm MDA-MB-231 cells (D) or hepatocytes and MCF7 co-cultures \pm NPCs (E). Graphs depict the concentrations of a total of 55 analytes evaluated by Luminex on $48 \mathrm{~h}$ Day 15 supernatants $(n=2$ donors). Significantly altered analytes: (C) IL-1 $\beta(P<0.01)$ and (D) EGF $(P<0.05)$, VEGF-A $(P<0.05)$, IGFBP-1 $(P<0.01)$, uPA $(P<0.01)$, sEGF $(P<0.05)$, follastatin $(P<0.05)$, G-CSF $(P<0.01)$, and osteopontin $(P=0.17)$. Statistical analyses are Student's t-tests comparing co-culture conditions.

of MDA-MB-231 cells, marked rises were observed in proinflammatory molecules (IGFBP-1, IL-8, IL-6) and known cancer associated factors (sHER-2, PAI-1, osteopontin, uPA, PLGF, sCD40L). These data highlight the ability of the system to analyze communication networks in the micrometastatic niche, and suggest biomarkers for further evaluation.

\section{DISCUSSION}

Metastatic disease is the primary cause of cancer mortality and studies indicate that breast cancer cell dissemination is an early event, often occurring before primary tumour detection (Husemann et al, 2008). Few current models of breast cancer, metastasis can demonstrate a spontaneously dormant phenotype (Khanna and Hunter, 2005; Fantozzi and Christofori, 2006; Ghajar et al, 2013). However, not only do all-human model systems need to be utilised, but they should also incorporate supporting stromal and immune cells that are integral to accurately recapitulating the requisite microenvironment to elucidate the pathways involved in dormancy. Metastases, particularly to the liver, are understudied but vitally important due to the liver's role in metabolising the therapeutic agents administered to cancer patients. Further, the liver is a common metastatic site for multiple cancers, including breast cancer. Thus, the results obtained have implications for cancers beyond breast cancer.

We have established an all-human hepatic microenvironment that can effectively recapitulate human breast cancer cell dormancy even through 15 days of culture. We verified liver tissue function using a 
series of clinical and well-established phenotypic assays. There was low donor-to-donor variability for all of these markers except fibrinogen. Additionally, NPCs did not enhance or inhibit any of the hepatocyte phenotypes assayed, as expected. Most importantly, the persistence of high CYP activities through the entire 2-week culture period shows strong functioning, with limited hepatocyte damage (low AST and ALT) or inflammation (low A1AT) at the 2-week time point. Furthermore, the hepatocytes in this niche were unaffected by the presence of breast cancer cells, maintaining the functionality, in particular CYP metabolising activity, which is necessary for testing the efficacy and metabolism of metastatic breast cancer therapies as well as potential toxicities. This is similar to early stages of metastatic seeding to the liver during which liver function assays remain within normal ranges. Growth attenuation was confirmed by the absence of Ki67 staining and EdU incorporation in a population of aggressive MDA-MB-231 cells. Similar growth attenuation marked by a low proliferative index has been demonstrated in patient metastases in several studies (Pantel et al, 1993; Chao et al, 2010; Bednarz-Knoll et al, 2011). While it is evident that lack of proliferation may indicate states in addition to dormancy, the fuller picture of dormancy and emergence can be demonstrated by the target cells re-entering the mitogenic cycle at a later time period. Experimental demonstration of such would require complex protocols and analyses that lie beyond the scope of the current communication.

The inclusion of NPCs allows for a better understanding of the complex human signalling pathways at work in the micrometastatic niche of the liver. Understanding these signalling pathways, particularly in a human context, is critical to dissecting the mechanisms of entrance and exit to dormancy. We are still working to determine the origin of many of these signalling cascades and further experimentation is underway to tease out more specifics of this critical microenvironmental signalling. Luminex studies verified the sustained presence and viability of NPCs in the hepatic tissue. Further investigations revealed a differential growth response of MCF7 and MDA-MB-231 cell lines in the presence of NPCs. This mimics the variability seen in patients and indicates that there are signalling cascades that may help in understanding the difference in cancer outcomes. Luminex analysis of supernatants also demonstrated that the inclusion of the full complement of NPCs altered soluble signalling factors involved in both the attenuation and progression of cancer. For MDA-MB-231 cells, NPCs predominantly perpetuated a quiescent, non-proliferative microenvironment via reduced levels of pro-inflammatory factors and cancer-associated signals. Elevated levels of signals that protect against uncontrolled cellular proliferation and promote cellular differentiation (e.g., follistatin) were also detected and are integral functions in tissue rebuilding and repair (Sengupta et al, 2014). Conversely for the MCF7 cells, elevations were detected in pro-inflammatory and cancer-associated signals otherwise not observed in MDA-MB-231 cultures with NPCs. These differences in MDA MB 231 and MCF7 signalling networks are likely the basis for the contrasting outgrowth response noted between the two breast cancer cell lines. This indicates a potential role of NPCs within the liver microenvironment in enabling the establishment of the dormant micrometastatic niche.

Investigations into cancer cell signalling within the microenvironment revealed active contributions by the cancer cells even when in a quiescent state. Known cancer-associated factors (sHER-2, PAI-1, osteopontin, uPA, PLGF, sCD40L) were elevated, further confirming that the attenuated cancer cell populations were indeed viable. Cancer cells were associated with increases in inflammatory cytokines and chemokines, possibly creating a pro-inflammatory microenvironment, which may enable their integration into the hepatic tissue via the disruption of hepatocyte connections, such as E-cadherin (Yates et al, 2007). This highlights the ability of the system to begin investigations into capturing and elucidating the communication networks within the metastatic microenvironment. In vivo these measurements are not possible as the micrometastases are not clinically detectable and too few are present even in animal models to truly detect this level of change in the microenvironment. Furthermore, this MPS will potentially provide a basis for the development of new therapies that act to manipulate the identified signalling networks and subsequently improve prognosis.

The impact of the metastatic microenvironment on the disseminated tumour cells (Gunasinghe et al, 2012) and the crosstalk between the liver NPCs and malignant cells are both well appreciated although understudied. Malignant cells can activate hepatic stellate cells and provide a proangiogenic microenvironment through induction of VEGF and large endothelial cell recruitment (Olaso et al, 2003). Recent work in 3D organotypic models provides exploratory evidence for the role of endothelial cells in tumour dormancy (specifically in the lung and the brain) (Ghajar et al, 2013) and begins to tease out the permissive and inhibitory milieu of the bone marrow (Marlow et al, 2013). Our model system for the hepatic niche moves beyond organotypic modelling into microphysiologic systems, providing the mechanical stresses and oxygenation that is so important to the liver. A full complement of hepatic cells provides a microenvironment that explores more than co-culture conditions.

This microphysiologic system, like any model system, has limitations. Many of the hepatocytes and NPCs we receive are from patients previously treated with chemotherapy for colorectal liver metastases, and thus may present some dysfunction. Previous work does demonstrate that hepatocytes previously exposed to chemotherapy maintain viability and function (Hewes et al, 2006), and our work herein shows expected functioning suggesting that prior chemotherapy is not detrimental. Further, the hepatic niche also operates as a part of a larger system in the body and as such is influenced by other organs and blood borne factors; these aspects are not captured herein in the present iteration. This system begins to recapitulate the microenvironment of the liver but will require additional inputs to more accurately recreate the array of stimuli received in patients. As a part of our research we are continuing to explore these questions through connection of this microphysiologic system with other systems such as the lung, gut, and immune systems. Additional work with circadian variations in hormones and nutrients is underway to begin to uncover the complex temporal and systemic effects of other organs and hormones on the liver burdened with micrometastases (Wheeler et al, 2013). Still, this microphysiologic system represents the most advanced all human organoid culture system to study tumour cell biology in the metastatic niche.

\section{ACKNOWLEDGEMENTS}

The authors thank other members of their laboratories for thoughtful discussions. The work described herein is being funded by grants from the NIH (1UH2TR000496-01), a VA Merit Award, and DARPA (BAA-11-73 Microphysiological Systems: W911NF-12-2-0039).

\section{CONFLICT OF INTEREST}

A Wells: Patent on LiverChip now being commercialised by $\mathrm{CN}$ Bio Innovations Ltd and L Griffith: Patent on LiverChip now being commercialised by $\mathrm{CN}$ Bio Innovations Ltd; consulting fees paid by Zyoxel Ltd in 2012 but no current relationship. The remaining authors declare no conflict of interest.

\section{REFERENCES}

Bednarz-Knoll N, Alix-Panabieres C, Pantel K (2011) Clinical relevance and biology of circulating tumor cells. Breast Cancer Res 13(6): e228. 
Bukholm IK, Nesland JM, Borresen-Dale AL (2000) Re-expression of E-cadherin, alpha-catenin and beta-catenin, but not of gamma-catenin, in metastatic tissue from breast cancer patients. J Pathol 190(1): 15-19.

Cameron MD, Schmidt EE, Kerkvliet N, Nadkarni KV, Morris VL, Groom AC, Chambers AF, MacDonald IC (2000) Temporal progression of metastasis in lung: cell survival, dormancy, and location dependence of metastatic inefficiency. Cancer Res 60(9): 2541-2546.

Chao Y, Wu Q, Acquafondata M, Dhir R, Wells A (2012) Partial mesenchymal to epithelial reverting transition in breast and prostate cancer metastases. Cancer Microenviron 5: 19-28.

Chao YL, Shepard CR, Wells A (2010) Breast carcinoma cells re-express E-cadherin during mesenchymal to epithelial reverting transition. Mol Cancer 9: e179.

Crispe IN (2009) The liver as a lymphoid organ. Annu Rev Immunol 27: 147-163.

Demicheli R, Miceli R, Moliterni A, Zambetti M, Hrushesky WJ, Retsky MW, Valagussa P (2005) Breast cancer recurrence dynamics following adjuvant CMF is consistent with tumor dormancy and mastectomy-driven acceleration of the metastatic process. Ann Oncol 16(9): 1449-1457.

Domenech M, Bjerregaard R, Bushman W, Beebe DJ (2012) Hedgehog signaling in myofibroblasts directly promotes prostate tumor cell growth. Integr Biol 4(2): 142-152.

Fantozzi A, Christofori G (2006) Mouse models of breast cancer metastasis. Breast Cancer Res 8(4): 212.

Fidler IJ (1970) Metastasis: quantitative analysis of distrubtion and fate of tumor emboli labeled with 125I-5-iodo-2'-deoxyuridine. J Natl cancer Inst 45: 773-782.

Fisher B, Anderson S, Bryant J, Margolese RG, Deutsch M, Fisher ER, Jeong JH, Wolmark N (2002) Twenty-year follow-up of a randomized trial comparing total mastectomy, lumpectomy, and lumpetomy plus irradiation for the treatment of invasive breast cancer. N Engl J Med 347(16): 1233-1241.

Ghajar CM, Peinado H, Mori H, Matel IR, Evason KJ, Brazier H, Almeida D, Koller A, Hajjar KA, DYR Stainier, Chen EI, Lyden D, Bissell MJ (2013) The perivascular niche regulates breast tumour dormancy. Nat Cell Biol 15: 807-817.

Gomez EW, Chen QK, Gjorevski N, Nelson CM (2010) Tissue geometry patterns epithelial-mesenchymal transition via intercellular mechanotransduction. J Cell Biochem 110(1): 44-51.

Gonzalez-Angulo AM, Morales-Vasquez F, Hortobagyi GN (2007) Overview of resistance to systemic therapy in patients with breast cancer. $A d v$ Exp Med Biol 608: 1-22.

Griffith LG, Wells A, Stolz D (2013) Engineering liver. Hepatology 60(4): 1426-1434.

Gunasinghe N, Wells A, Thompson E, Hugo H (2012) Mesenchymalepithelial transition (MET) as a mechanism for metastatic colonisation in breast cancer. Cancer Metastasis Rev 31: 469-478.

Hackam DG, Redelmeier DA (2006) Translation of research evidence from animals to humans. JAMA 296(14): 1731-1732.

Hewes JC, Riddy D, Morris RW, Woodrooffe AJ, Davidson BR, Fuller B (2006) A prospective study of isolated human hepatocyte function following liver resection for colorectal liver metastases: the effects of prior exposure to chemotherapy. J Hepatol 45(2): 263-270.

Husemann Y, Geigl JB, Schubert F, Musiani P, Meyer M, Burghart E, Forni G, Eils R, Fehm T, Riethmuller G, Klein CA (2008) Systemic spread is an early step in breast cancer. Cancer Cell 13(1): 58-68.

Hwa AJ, Fry RC, Sivaraman A, So PT, Samson LD, Stolz DB, Griffith LG (2007) Rat liver sinusoidal endothelial cells survive without exogenous VEGF in 3D perfused co-cultures with hepatocytes. FASEB J 21(10): 2564-2579.

Junttila MR, de Sauvage FJ (2013) Influence of tumour micro-environment heterogeneity on therapeutic response. Nature 501(7467): 346-354.

Khanna C, Hunter K (2005) Modeling metastasis in vivo. Carcinogenesis 26(3): 513-523.

Kowalski PJ, Rubin MA, Kleer CG (2003) E-cadherin expression in primary carcinoma of the breast and its distant metastases. Breast Cancer Res 5(6): R217-R222.

Luzzi KJ, MacDonald IC, Schmidt EE, Kerkvliet N, Morris VL, Chambers AF, Groom AC (1998) Multistep nature of metastatic inefficiency: dormancy of solitary cells after successful extravasation and limited survival of early micrometastases. Am J Pathol 153(3): 865-873.

Marlow R, Honeth G, Lombardi S, Cariati M, Hessey S, Pipili A, Mariotti V, Buchupalli B, Foster K, Bonnet D, Grigoriadis A, Rameshwar P, Purushotham A, Tutt A, Dontu G (2013) A novel model of dormancy for bone metastatic breast cancer cells. Cancer Res 73(23): 6886-6899.
Mestas J, Hughes CC (2004) Of mice and not men: differences between mouse and human immunology. J Immunol 172(5): 2731-2738.

Micalizzi DS, Farabaugh SM, Ford HL (2010) Epithelial-mesenchymal transition in cancer: parallels between normal development and tumor progression. J Mammary Gland Biol Neoplasia 15(2): 117-134.

Nelson CM, Vanduijn MM, Inman JL, Fletcher DA, Bissell MJ (2006) Tissue geometry determines sites of mammary branching morphogenesis in organotypic cultures. Science 314(5797): 298-300.

Olaso E, Salado C, Egilegor E, Gutierrez V, Santisteban A, Sancho-Bru P, Friedman SL, Vidal-Vanaclocha F (2003) Proangiogenic role of tumoractivated hepatic stellate cells in experimental melanoma metastasis. Hepatology (Baltimore, MD) 37(3): 674-685.

Pantel K, Schlimok G, Braun S, Kutter D, Lindemann F, Schaller G, Funke I, Izbicki JR, Riethmuller G (1993) Differential expression of proliferationassociated molecules in individual micrometastatic carcinoma cells. J Natl Cancer Inst 85(17): 1419-1424.

Perloff M, Hart RD, Holland JF (1978) Vinblastine, adriamycin, thiotepa, and halotestin (VATH): therapy for advanced breast cancer refractory to prior chemotherapy. Cancer 42(6): 2534-2537.

Pillai VC, Strom SC, Caritis SN, Venkataramanan R (2013) A sensitive and specific CYP cocktail assay for the simultaneous assessment of human cytochrome P450 activities in primary cultures of human hepatocytes using LC-MS/MS. J Pharm Biomed Anal 74: 126-132.

Powers MJ, Domansky K, Capitano A, Kalezi A, Udapadhaya A, Kaazempur-Mofrad MR, Kurzawski P, Wack KE, Stolz DB, Kamm R, Griffith LG (2002) A microarray perfusion bioreactor for 3D liver culture. Biotechnol Bioeng 78(3): 257-269.

Ridky TW, Chow JM, Wong DJ, Khavari PA (2010) Invasive threedimensional organotypic neoplasia from multiple normal human epithelia. Nat Med 16(12): 1450-1455.

Sengupta D, Bhargava DK, Dixit A, Sahoo BS, Biswas S, Biswas G, Mishra SK (2014) ERRbeta signalling through FST and BCAS2 inhibits cellular proliferation in breast cancer cells. Br J Cancer 110(8): 2144-2158.

Shaw KR, Wrobel CN, Brugge JS (2004) Use of three-dimensional basement membrane cultures to model oncogene-induced changes in mammary epithelial morphogenesis. J Mammary Gland Biol Neoplasia 9(4): 297-310.

Slaney CY, Rautela J, Parker BS (2013) The emerging role of immunosurveillance in dictating metastatic spread in breast cancer. Cancer Res 73(19): 5852-5857.

Stroock AD, Fischbach C (2010) Microfluidic culture models of tumor angiogenesis. Tissue Eng Part A 16(7): 2143-2146.

Taylor DP, Wells JZ, Savol A, Chennubhotla C, Wells A (2013) Modeling boundary conditions for balanced proliferation in metastatic latency. Clin Cancer Res 19(5): 1063-1070.

Teicher BA (2006) Tumor models for efficacy determination. Mol Cancer Ther 5(10): 2435-2443.

Viadana E, Bross ID, Pickren JW (1973) An autopsy study of some routes of dissemination of cancer of the breast. Br J Cancer 27(4): 336-340.

Weigelt B, Ghajar CM, Bissell MJ (2014) The need for complex 3D culture models to unravel novel pathways and identify accurate biomarkers in breast cancer. Adv Drug Deliv Rev 69-70: 42-51.

Wells A, Griffith L, Wells JZ, Taylor DP (2013) The dormancy dilemna: quiescence versus balanced proliferation. Cancer Res 73: 3811-3816.

Wheeler S, Borenstein J, Clark AM, Ebrahimkhani M, Fox IJ, Griffith L, Inman W, Lauffenburger DA, Nguyen T, Pillai VC, Prantil-Baun R, Stolz DB, Taylor DP, Ulrich T, Venkataramanan R, Wells A, Young C (2013) All-Human Microphysical Model of Metastasis Therapy. Stem Cell Res Ther 4(Suppl 1): S11.

Yates C, Shepard CR, Papworth G, Dash A, Beer Stolz D, Tannenbaum S, Griffith L, Wells A (2007) Novel three-dimensional organotypic liver bioreactor to directly visualize early events in metastatic progression. Adv Cancer Res 97: 225-246.

This work is published under the standard license to publish agreement. After 12 months the work will become freely available and the license terms will switch to a Creative Commons AttributionNonCommercial-Share Alike 3.0 Unported License.

Supplementary Information accompanies this paper on British Journal of Cancer website (http://www.nature.com/bjc) 